\title{
The effectiveness of surgical interventions for women with FGM/C: a systematic review
}

\author{
RC Berg, ${ }^{a, b, \dagger}$ S Taraldsen, ${ }^{c, \dagger}$ MA Said, ${ }^{d}$ IK Sørbye, ${ }^{c}$ S Vangen ${ }^{c, e}$ \\ ${ }^{a}$ Norwegian Institute of Public Health, Oslo, Norway ${ }^{b}$ Department of Community Medicine, University of Tromso, Tromso, Norway \\ ${ }^{\mathrm{c}}$ Norwegian National Advisory Unit on Women's Health, Division of Gynaecology and Obstetrics, Oslo University Hospital HF \\ Rikshospitalet, Oslo, Norway ${ }^{\mathrm{d}}$ Oslo University Hospital - Ullevål Hospital, Oslo, Norway ${ }^{\mathrm{e}}$ Institute of Clinical Medicine, University of Oslo, \\ Oslo, Norway \\ Correspondence: Dr RC Berg, Norwegian Institute of Public Health, PO Box 4404, Nydalen, N-0403 Oslo, Norway. Email rigmor.berg@fhi.no
}

Accepted 4 July 2017. Published Online 28 August 2017.

This article includes Author Insights, a video abstract available at https://vimeo.com/rcog/authorinsights14839.

Background Female genital mutilation/cutting (FGM/C) changes normal genital functionality and can cause complications. There is an increasing demand for treatment of FGM/C-related complications.

Objectives We conducted a systematic review of empirical quantitative research on the outcomes of interventions for women with FGM/C-related complications.

Search strategy A search specialist searched 16 electronic databases.

Selection criteria Selection was performed independently by two researchers. We accepted quantitative studies that examined the outcome of an intervention for an FGM/C-related concern.

Data collection and analysis We extracted data into a predesigned form, calculated effect estimates, and performed metaanalyses.

Main results We included 62 studies (5829 women), which investigated the effect of defibulation, excision of cysts, and clitoral reconstruction. Meta-analyses of defibulation versus no defibulation showed a significantly lower risk of caesarean section (relative risk, RR: 0.33 ; 95\% confidence interval, 95\% CI: $0.25-0.45)$ and perineal tears with defibulation: second-degree tear (RR: $0.44,95 \%$ CI:
0.24-0.79), third-degree tear (RR: $0.21,95 \%$ CI: $0.05-0.94$ ), fourth-degree tear (RR: 0.06, 95\% CI: 0.01-0.41). The metaanalyses detected no significant differences in obstetric outcomes of antenatal versus intrapartum defibulation. Except for one study, none of the studies on the excision of cysts indicated any complications, and the results were deemed favourable. Reconstructive surgery resulted in a visible clitoris in about $77 \%$ of women. Most women self-reported improvements in their sexual life, but up to $22 \%$ experienced a worsening in sexuality-related outcomes after reconstruction.

Conclusions Women with FGM/C who seek therapeutic surgery should be informed about the scarcity of evidence for benefits and the potential harms of the available procedures.

Keywords Defibulation, excision of cyst, female genital mutilation/cutting, obstetric, reconstructive surgery, systematic review.

Tweetable abstract Systematic review shows defibulation after FGM/C has obstetric benefits; effect of reconstruction is uncertain.

Linked article This article is commented on by M McCauley, p. 288 in this issue. To view this mini commentary visit https:// doi.org/10.1111/1471-0528.14925.

Please cite this paper as: Berg RC, Taraldsen S, Said MA, Sørbye IK, Vangen S. The effectiveness of surgical interventions for women with FGM/C: a systematic review. BJOG 2018;125:278-287.

\section{Introduction}

Female genital mutilation/cutting (FGM/C) is a term that encompasses a variety of procedures performed on the external female genitalia for cultural or other non-

†Shared first authorship. therapeutic reasons. The World Health Organization (WHO) classification describes four types: clitoridectomy (type I), excision (type II), infibulation (type III), and type IV, which describes all other harmful procedures to the female external genital tissue, such as pricking and cauterising. ${ }^{1}$ These procedures change normal genital functionality. Consequently, many women experience both immediate 
and lifelong complications. Systematic reviews reveal that the most common immediate complications following FGM/C include excessive bleeding, pain, genital tissue swelling, and urine retention, ${ }^{2}$ whereas in the long-term, women living with FGM/C have an increased risk of gynaecological, sexual, and obstetric complications..$^{2-4}$ In particular, meta-analyses show that women with FGM/C are at greater risk of prolonged labour, obstetric lacerations, instrumental delivery, difficult delivery, and obstetric haemorrhage. ${ }^{4}$ A modelling analysis found that across six African countries alone, an estimated 130000 life years were lost owing to obstetric haemorrhage associated with FGM/C. ${ }^{5}$

Concurrent with the adverse health effects, there is an increasing demand for FGM/C-related treatments and, in turn, recognition for the need to build the capacity to enable healthcare providers to manage FGM/C-related complications. Across 15 countries in Africa where the practice is concentrated, more than 5500 health facilities have recently integrated $\mathrm{FGM} / \mathrm{C}$ in their antenatal and postnatal care services. ${ }^{6}$ In infibulated women, defibulation is considered necessary prior to childbirth. This is a minor surgical procedure, involving an anterior incision of the infibulated scar tissue, which re-opens the vaginal introitus and creates new labia majora. ${ }^{7}$ In Western countries, specialisation units for women with FGM/C are appearing, ${ }^{8-10}$ including options for surgical treatments that aim to reconstruct the clitoral and labial tissues of women with FGM/ C. ${ }^{11}$ In general, such surgical treatments intend to create a genital appearance similar to women without FGM/C, manage complications, improve functionality, and prevent obstetric complications.

Despite recent reviews and guidelines, ${ }^{7,12}$ the effectiveness of interventions to manage FGM/C-related complications remain uncertain. There are discrepancies among experts' opinions, guidelines, and national health policies. $^{7,13,14}$ Our study objective was to summarise the empirical quantitative research on the immediate and long-term outcomes of treatment interventions for women with FGM/C-related complications.

\section{Methods}

The present systematic review followed the guidelines in the Cochrane Handbook for Systematic Reviews of Interventions. ${ }^{15}$ We included research that reported on outcomes associated with any treatment intervention for women with an FGM/C-related problem. Specifically, we accepted all quantitative study designs, with females of any age and nationality who had been subjected to any type of FGM/C, according to the WHO modified typology, ${ }^{1}$ that examined the outcome of a treatment intervention for an FGM/Crelated concern. Eligible comparisons were women who have undergone FGM/C but have not received any intervention, or have received a different intervention. We included all types of outcomes: both short- and long-term benefits and harms experienced by women, or their infants. We had no language limitations and included all relevant studies, irrespective of publication format.

A search specialist designed and performed electronic database searches in 16 electronic databases for the identification of studies published from 1980 through to January 2012. We updated the search in May 2016 for the six databases that provided the highest and most relevant yield: EMBASE, MEDLINE, MEDLINE In-Process \& Other NonIndexed Citations, PILOTS, POPLINE, and PsycINFO. All databases and the complete search strategy for each are shown in Appendix S1. Complementary methods to identify studies included following up on citations, hand searching, and scanning the reference lists of relevant papers.

Following the searches, all studies considered pertinent on the basis of the title and abstract were retrieved. Subsequently, the same researchers independently read the full text to verify the pertinence of the study to the systematic review. Upon inclusion, one researcher extracted data [population, intervention, comparison, outcome (PICO) and outcomes] onto a pre-designed form and a second researcher checked for accuracy. Disagreements between researchers were resolved by mutual discussion. Methodological quality assessments (risk of bias) were undertaken as recommended in the Cochrane handbook, ${ }^{15}$ using design-specific checklists based on the user's guide framework. ${ }^{16}$ When necessary, we contacted the study authors to obtain further information.

We pooled data using Review Manager (REvMan 5.3) (The Cochrane Collaboration, The Nordic Cochrane Centre, Copenhagen, Denmark) when studies were conceptually similar and extractable data were available. Dichotomous outcomes were expressed as a risk ratio (RR) with a $95 \%$ confidence interval (95\% CI) using a randomeffect model. Heterogeneity between studies was based on the results of the $I^{2}$ and $\chi^{2}$ statistics. High heterogeneity was defined as $I^{2}>50 \%$ or $P<0.10$. A value of $P<0.05$ was considered to be statistically significant. Finally, we applied the instrument Grading of Recommendations Assessment, Development and Evaluation (GRADE) with GRADE-PROFILER 3.6 [GRADEpro Guideline Development Tool (software) (developed by Evidence Prime, Inc.); McMaster University, Hamilton, Canada] to assess the extent to which we can have confidence in the effect estimates (gradeworkinggroup.org). ${ }^{17}$ We used the standard definitions for grading the quality of the evidence: high, moderate, low, and very low. ${ }^{18}$

The systematic review was registered in the PROSPERO International Prospective Register of Systematic Reviews (reg. no. CRD4201501985; 24 April 2015). The Preferred Reporting Item for Systematic Reviews and Meta-analysis was followed. The study had no specific funding. 
Berg et al.

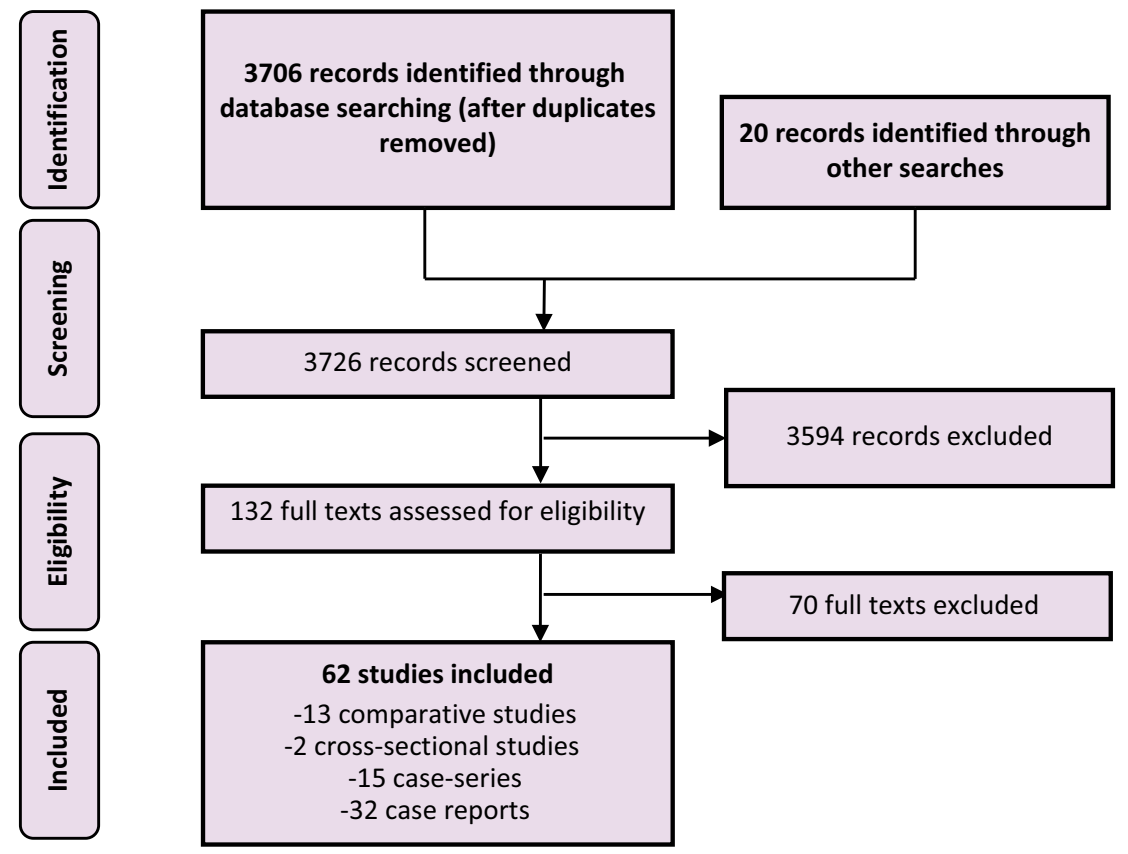

Figure 1. PRISMA flow diagram of literature reviewing process.

\section{Results}

A total of 3726 unique citations were identified. Of these, a total of 62 studies (denoted $k$ ) were included (Figure 1). Details of all included studies are shown in Tables S1 and S2, along with 70 excluded studies screened in full text (Table S3). For transparency, figures $(k)$ in the text represent the number of included studies reporting the respective findings.

In total, the 62 studies included 5829 women (denoted $n)$. In this communication, in line with recommendations, ${ }^{15}$ we primarily present the results from the studies deemed to have the highest internal validity with respect to the question of benefit and harm. These were 13 comparative studies with 4743 participants: four retrospective case series with two or more groups, ${ }^{19-22}$ two prospective cohort studies with two or more groups, ${ }^{23,24}$ one controlled before-and-after study, ${ }^{25}$ and six uncontrolled beforeand-after studies. ${ }^{13,26-30}$ The remaining 49 studies were non-comparative studies (case reports, case series, crosssectional studies; Table S1). The methodological study quality was variable, with a minority of studies (16.2\%) assessed as having high methodological quality (Table S4).

Although the setting was primarily Europe and the USA $(k=37)$, all women in the included studies originated from a country where FGM/C is commonly practiced. The majority (70\%) had FGM/C type III, whereas almost all the other women had type II (28\%).

Three types of interventions were identified, all surgical: defibulation/surgical separation of fused labia; excision of cysts (generally with some form of reconstruction of the clitoris and/or labia); and clitoral/clitorolabial reconstructive surgery. These studies differed in respect to their objectives. The 32 studies on defibulation primarily focused on obstetrics-related outcomes. The 21 studies, mostly case reports, addressing the excision of cysts almost all dealt with intra- and postoperative complications (most data from these studies are relegated to Table S1). The nine studies addressing reconstructive surgery concerned mainly sexuality-related outcomes.

\section{Defibulation}

Five studies reported on intraoperative complications, finding that there were no complications among 436 women. Postoperative complications occurred among 14/113 $(12.4 \%)$ women $(k=7)$, and were of minor character, such as urinary tract infections, wound infections, and labial adhesions. Recovery and healing were satisfactory at 13 months of follow-up, with good anatomical results $(k=13, n=117)$.

Twelve studies $(n=191)$ reported on outcomes related to sexual function. The before-and-after study $(n=18)$ conducted by Krause et al. ${ }^{28}$ on sexual functioning-measured with the validated Female Sexual Function Index (FSFI, range 2-36, with lower scores indicating more problematic sexual function) - was the most informative. It found that sexual functioning improved in all domains at 6 months follow-up after defibulation: desire $(4.1 \rightarrow 5.0)$, arousal $(0.9 \rightarrow 1.5)$, lubrication $(2.3 \rightarrow 2.5)$, orgasm $(1.3 \rightarrow 1.8), \quad$ satisfaction $\quad(1.3 \rightarrow 2.4), \quad$ and pain 
$(0.5 \rightarrow 5.8)$. The improvement was not significant in the domains of lubrication and orgasm. Similarly, women $(n=48)$ in Hussen and Catania's study reported that after defibulation, sex was less painful $(73.3 \%)$ and that sex improved to the point that they could reach orgasm (93.8\%), but $6.3 \%$ experienced no improvement in sexual function. ${ }^{28}$ Three other studies $(n=63)$ reported similar positive results on sexual function. ${ }^{31-33}$

Eight studies $(n=2069)$ reported on more than 30 different outcomes concerning obstetrics (Tables S5 and S6). As seen in Table S5, the five studies that examined defibulation versus no defibulation measured few identical outcomes, and found few significant differences between the groups. In two studies the no-defibulation group consisted of women with FGM/C types I and II, ${ }^{19,24}$ and thus these studies were not included in the meta-analyses. The metaanalyses indicated statistically significant differences in caesarean section, second-degree tear, third-degree tear, and fourth-degree tear (Figure 2). Women with defibulation had a lower risk of caesarean section (RR: 0.33, 95\% CI: 0.25-0.45) and perineal tears: second-degree tear (RR: 0.44, 95\% CI: 0.24-0.79), third-degree tear (RR: 0.21, 95\% CI: 0.05-0.94), and fourth-degree tear (RR: 0.06, 95\% CI: $0.01-0.41)$.

Four studies examined antenatal defibulation versus intrapartum defibulation on a range of outcomes, which they often measured differently (Table S6). Results varied considerably across the studies, including the direction of results. The meta-analyses detected no statistically significant differences in the risk of caesarean section (RR: 1.55, 95\% CI: 0.56-4.30) and episiotomy (RR: 1.18, 95\% CI: $0.32-4.35$ ) relative to the timing of defibulation (Figure 3). Our meta-analysis of two studies on the effect of previous (prior to pregnancy or during previous pregnancy) versus antenatal defibulation on caesarean section showed no statistically significant difference (RR: $0.91,95 \%$ CI: $0.50-$ 1.64; Figure S1). ${ }^{20,21}$

\section{Excision of cysts}

There were no intraoperative complications reported from the excision of cysts $(k=13, n=91)$, bleeding was minimal (about $100 \mathrm{ml}$ ), and women recovered well $(k=4$, $n=47)$. There were no recurrences of cysts at 1-6 years of follow-up $(k=6, n=97)$, the anatomical appearance was good at 1-7 months of follow-up $(k=4, n=4)$, and women's sexual life had improved at 1-7 months of followup $(k=6, n=49)$. The results of the controlled beforeand-after study from Egypt are worth highlighting. ${ }^{25}$ It described the sex scores-measured with the non-validated Kasr El Aini sexual assessment questionnaire (range 1-100, with a higher score indicating better sexual function) - of three groups of women $(n=87)$ who all received a different type of intervention. There was a change in the scores from before to after the operation at 6 months for all three groups: clitorolabial reconstruction $(65.6 \rightarrow 80.5)$, excision of cysts and clitorolabial reconstruction $(76.9 \rightarrow 79.0)$, and excision of cysts $(76.7 \rightarrow 63.0)$. Thus, the two groups that received clitorolabial reconstruction improved, whereas the women who only received the excision of cysts experienced a worsening of their sex score. The mean postoperative difference between the two groups of women who all had cysts was 16.00 (SD: 15.43-16.57, $P<0.001$ ).

\section{Reconstructive surgery}

Four studies ( $n=3515)$ examined postoperative complications from clitoral reconstruction (Table S7). On average, $5.3 \%$ experienced immediate complications and $15.4 \%$ experienced minor postoperative complications at 810 days of follow-up, such as haematoma, infections, and suture failure. Eight studies $(n=3725)$ reported on the outcomes of clitoral reconstruction. Table 1 details results for the sexuality-related outcomes at follow-up, which varied among the included studies (results of the three case reports are not tabled). ${ }^{34-36}$ With respect to genital appearance, reconstructive surgery resulted in a visible clitoris for an average of $77.5 \%$ of the women (range $69.7-100 \%$ ). On the whole, a substantial proportion of women self-reported improvements in their sexual life 1-12 months after reconstruction. There were improvements regarding pain during sex (average 43\%, range 8-76.9\%), clitoral function/pleasure $(63 \%$, range $60.7-88.5 \%)$, desire $(50.8 \%$, range $46.8-$ $55.7 \%)$, and frequency of sex $(41 \%)$. One before-and-after study $(n=61)$ found that reconstruction resolved bleeding during sex for $15 \%$ of the women, vaginismus/dryness for $26 \%$ of the women, and the involuntary passing of urine during sex for $5 \%$ of the women. ${ }^{29}$ Similarly, as assessed by the Kasr El Aini sexual assessment questionnaire, women who received clitorolabial reconstruction $(n=30)$ reported improvement at 6 months after the operation (from 65.6 to 80.5$).^{25}$

Conversely, as seen in Table 1, although few studies assessed any harm from reconstruction, the results indicated that $2.3 \%$ of women experienced a worsening in clitoral pleasure, $8.2 \%$ decreased desire, $8.2 \%$ decreased frequency of sex, $2.0 \%$ recounted genital pain that was not present before surgery, and $22.6 \%$ of women who had regular orgasm prior to surgery reported restricted orgasm after surgery. Non-clinical outcomes were reported in one study. Abramowicz et al. ${ }^{37}$ found that among 26 women followed up 6-12 months after clitoral reconstruction, 88-96\% perceived an improvement in body image, genital image, relationship with others, psychological well-being, and aesthetic appearance. None of the studies on clitoral reconstruction reported outcomes concerning obstetrics or other outcomes. We note that not all outcomes from each study are communicated here, because of space limitations and irregular 
Berg et al.

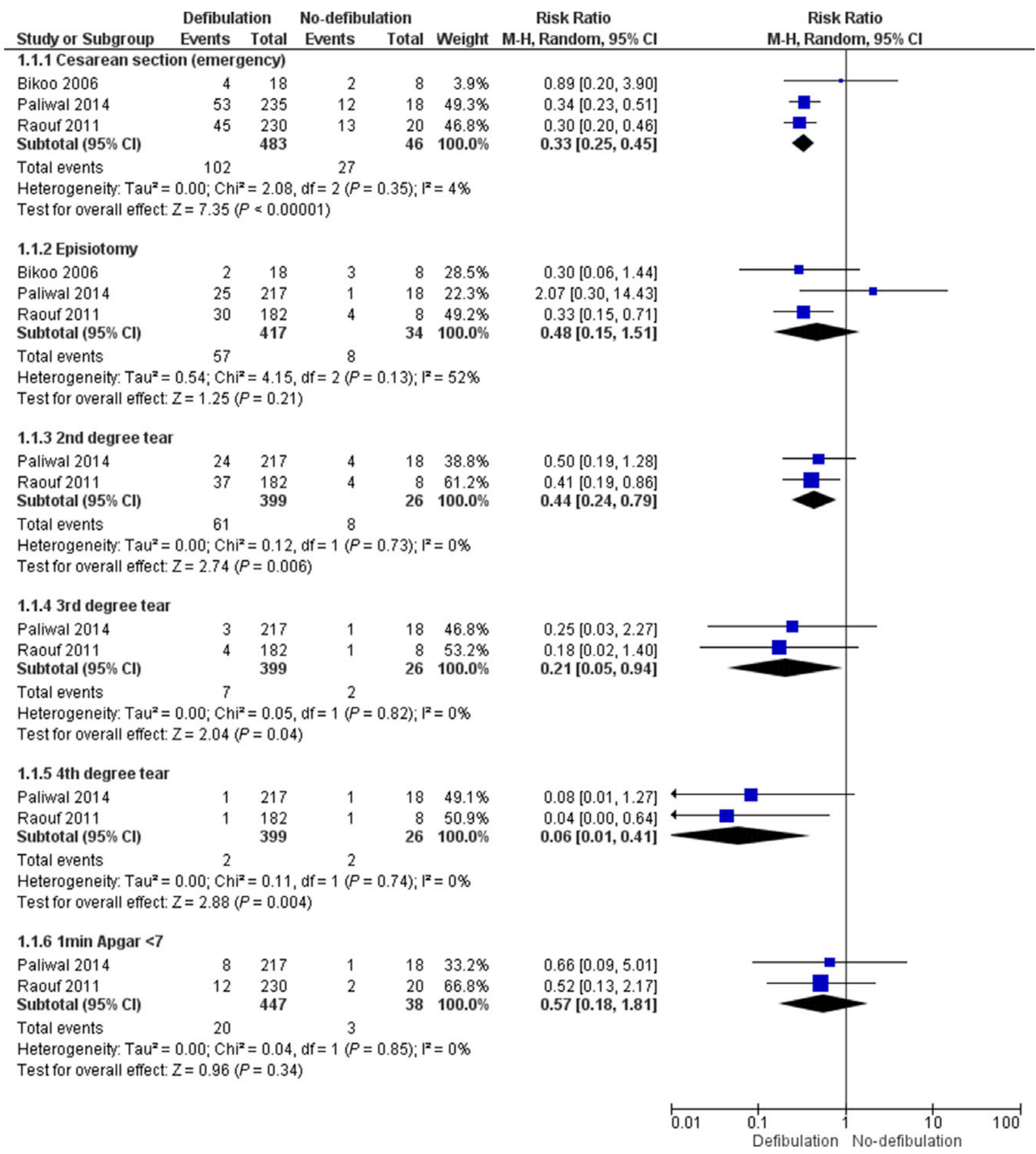

Figure 2. Meta-analyses of defibulation versus no defibulation, for caesarean section, episiotomy, tears, and Apgar score.

study reporting. For example, in the study by Foldès et al. ${ }^{13}$ the team operated on 2939 women, reported follow-up data for 866 women $(29.5 \%)$ at 1 year, and presented dissimilar pre- and post-operation outcome categories.
We judged that there is very low confidence in the effect estimates for all outcomes, because of the observational study designs and a combination of risk of bias, imprecision, and/or inconsistency (Tables S8-S10). 
Antepartum defib Intrapartum defib

Risk Ratio

Risk Ratio

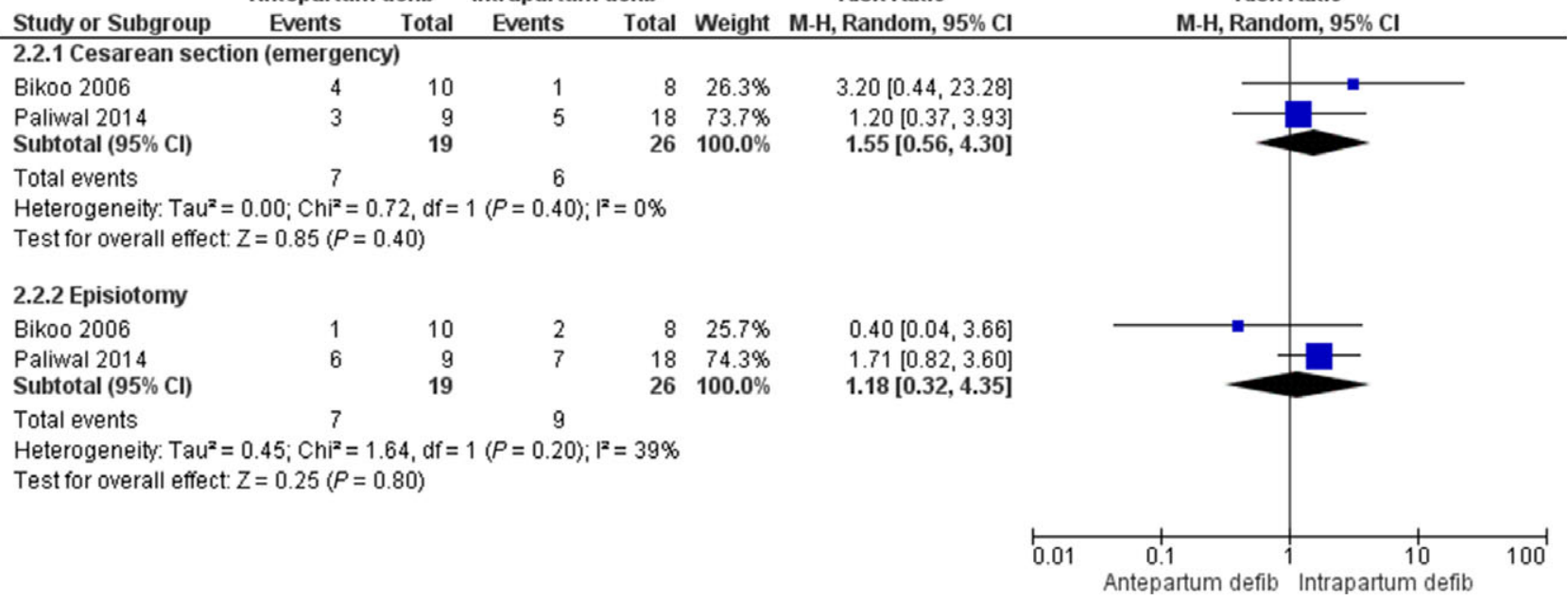

Figure 3. Meta-analyses of antepartum defibulation versus intrapartum defibulation, for caesarean section and episiotomy.

Table 1. Study outcomes and effect estimates of reconstruction for women with FGM/C (outcomes: sexual function)

\begin{tabular}{|c|c|c|c|c|c|c|}
\hline \multirow{2}{*}{$\begin{array}{l}\text { Study } \\
\text { Pain during intercours }\end{array}$} & \multirow[t]{2}{*}{ Follow-up } & \multirow[t]{2}{*}{ Outcome } & \multicolumn{2}{|c|}{ Rate pre-surgery } & \multicolumn{2}{|c|}{ Rate post-surgery } \\
\hline & & & & & & \\
\hline Abramowicz $(2016)^{37}$ & 6 months & Improvement of pain & - & & $20 / 26$ & $76.9 \%$ \\
\hline Foldès $(2006)^{26}$ & 6 months & Discomfort during sex & $97 / 453$ & $21.4 \%$ & $13 / 97$ & $13.4 \%$ \\
\hline Foldès $(2012)^{13}$ & 1 year & Improvement in dyspareunia & - & & 99/202 & $49.0 \%$ \\
\hline Merckelbagh $(2015)^{29}$ & 1 month & Dyspareunia & $29 / 61$ & $47.5 \%$ & $5 / 61$ & $8.2 \%$ \\
\hline Ouedraogo $(2013)^{30}$ & 6 months & Dyspareunia & $37 / 94$ & $39.4 \%$ & $1 / 94$ & $1.1 \%$ \\
\hline \multicolumn{7}{|l|}{ Sexual function } \\
\hline \multirow[t]{3}{*}{ Abramowicz $(2016)^{37}$} & 6 months & Improvement in clitoral function & - & & $23 / 26$ & $88.5 \%$ \\
\hline & & Improvement in desire & - & & $14 / 26$ & $53.8 \%$ \\
\hline & & Improvement in satisfaction & - & & $19 / 26$ & $73.1 \%$ \\
\hline \multirow[t]{3}{*}{ Foldès $(2006)^{26}$} & 6 months & Normal clitoral sexuality & - & & $65 / 453$ & $14.3 \%$ \\
\hline & & Real improvement, no orgasm & - & & $146 / 453$ & $32.2 \%$ \\
\hline & & Never pleasure & $173 / 453$ & $38.2 \%$ & $1 / 453$ & $0.2 \%$ \\
\hline \multirow[t]{4}{*}{ Foldès $(2012)^{13}$} & 1 year & Improvement in clitoral pleasure & - & & $224 / 368$ & $60.7 \%$ \\
\hline & & Orgasm & - & & $280 / 684$ & $40.9 \%$ \\
\hline & & Worsening orgasm & - & & $12 / 53$ & $22.6 \%$ \\
\hline & & Worsening clitoral pleasure & - & & $20 / 866$ & $2.3 \%$ \\
\hline \multirow[t]{9}{*}{ Merckelbagh $(2015)^{29}$} & 1 month & Good clitoral sensation & $9 / 61$ & $9.8 \%$ & $36 / 61$ & $59.0 \%$ \\
\hline & & Orgasm & $1 / 61$ & $1.6 \%$ & $10 / 61$ & $16.4 \%$ \\
\hline & & Increased desire & - & & $34 / 61$ & $55.7 \%$ \\
\hline & & Decreased desire & - & & $5 / 61$ & $8.2 \%$ \\
\hline & & Increased frequency of sex & - & & $25 / 61$ & $41.0 \%$ \\
\hline & & Decreased frequency of sex & - & & $5 / 61$ & $8.2 \%$ \\
\hline & & Bleeding during intercourse & $10 / 61$ & $16.4 \%$ & $1 / 61$ & $1.6 \%$ \\
\hline & & Vaginismus/dryness & $27 / 61$ & $44.2 \%$ & $11 / 61$ & $18.0 \%$ \\
\hline & & Cystitis/vaginitis & $9 / 61$ & $14.8 \%$ & $4 / 61$ & $6.6 \%$ \\
\hline \multirow[t]{5}{*}{ Ouedraogo $(2013)^{30}$} & 6 months & Often desire & $38 / 94$ & $40.4 \%$ & $49 / 94$ & $52.1 \%$ \\
\hline & & Never desire & $39 / 94$ & $41.5 \%$ & $5 / 94$ & $5.3 \%$ \\
\hline & & Normal clitoral sexuality & - & & $36 / 94$ & $38.3 \%$ \\
\hline & & Real improvement, no orgasm & - & & $14 / 94$ & $14.9 \%$ \\
\hline & & Orgasm & $12 / 94$ & $12.8 \%$ & $34 / 94$ & $36.1 \%$ \\
\hline
\end{tabular}




\section{Discussion}

This systematic review synthesised the body of empirical effectiveness research on treatments for women with FGM/ C. The results can inform guidelines on treatment, help develop treatment services for women with FGM/C, and guide future research.

\section{Main findings}

The review identified little empirical evidence on benefits and harms from therapeutic surgeries for women with FGM/C, and no studies on other types of interventions. Only 13 comparative studies have examined this issue, many are retrospective, and all have methodological shortcomings. Bearing these weaknesses in mind, there is a growing body of evidence on a wide range of immediate and short-term effects of defibulation on obstetric outcomes, mostly for the mother but also for the infant. Regrettably, because of study heterogeneity, few meta-analyses were possible. We detected no statistically significant differences between antenatal defibulation and intrapartum defibulation, but found a favourable effect of defibulation relative to none with respect to caesarean section and perineal tears. We note that none of the studies, except one, ${ }^{24}$ stratified or controlled for parity (or other potential confounding factors, such as anaesthesia), and thus these results should be interpreted with caution. There is very little evidence on the immediate, short-, and long-term effects of defibulation on other health outcomes. Data from a few hundred women indicate that sexual functioning in areas such as pain improves. It is important to keep in mind that, despite the lack of evidence, functionally, by opening the introitus, defibulation facilitates sexual intercourse and restores ordinary physiological functions such as unobstructed urination in women who live with FGM/C type III.

Although there is a larger body of evidence on reconstructive surgery relative to defibulation, the effect of reconstruction is more uncertain, because of methodological limitations and insufficient study similarity to allow for pooling of effect estimates. Generally speaking, however, the results suggest that about three-quarters of women regain a visible clitoris. Self-reported improvements in pain during sex, clitoral function/pleasure, orgasm, and desire are in the $43-63 \%$ range, but up to $22 \%$ experience a worsening in sexuality-related outcomes. As with defibulation, there is a lack of evidence on the effects of reconstruction on other health outcomes. Data are sparse with respect to the excision of cysts, in particular, but one controlled before-and-after study suggests a worsening in sexual function. ${ }^{25}$ Caution is advised regarding surgery undertaken for cysts in the clitoral area.

\section{Strengths and limitations}

The strength of the systematic review lies in the rigorous methodology employed. We are confident that we have included all relevant studies and presented the most up-todate, comprehensive, and unbiased assessment possible. Nonetheless, we are cognizant that new studies are published quite regularly. Our last search was in May 2016, and we are aware that there is at least one ongoing beforeand-after study on clitoris reconstruction. ${ }^{38}$ It is also possible that different researchers may interpret what counts as a treatment for FGM/C-related complications slightly differently, but it is a strength that we employed broad inclusion criteria. As all reviewers, however, we were limited by what is reported, and in many cases, data extraction was challenging because of poor reporting in the included studies.

\section{Interpretation}

Few attempts at synthesising the evidence on the effect of interventions for women with an FGM/C-related concern exist. One review, published in the Cochrane library, addressed the effect of interventions to improve outcomes in pregnant women who have undergone FGM/C. The inclusion criteria stipulated that only randomised controlled trials (RCTs), cluster-, and quasi-RCTs were eligible, and no studies met the inclusion criteria. ${ }^{39}$ Our literature search, undertaken 4 years later, confirmed the lack of rigorous study designs with respect to interventions in not just pregnant women but in any women who have undergone FGM/C. Another review addressed safety and efficacy of clitoral reconstruction in women with FGM/C. It described the results of four studies and concluded that additional research was needed. ${ }^{12}$ Our findings concur with these reviewers' call for additional research.

Over the years, various guidelines have been published on how to treat women with FGM/C. ${ }^{7,40-42}$ They generally recommend that defibulation is performed for pregnant women who have undergone FGM/C type III. Our results indicate that there may be obstetric advantages of defibulation over no defibulation, but there is a scarcity of research on other outcomes. This is paradoxical, given that sexuality-related concerns and voiding and menstruation difficulties are the most common reasons for women seeking defibulation. ${ }^{43}$

The latest guideline on the management of complications from FGM/C, developed by the WHO, recommends undertaking defibulation in the antepartum or intrapartum period. ${ }^{7}$ This differs somewhat from the guidelines of three other organisations, which recommend defibulation before first sexual intercourse or pregnancy, intrapartum, and before pregnancy, respectively. ${ }^{40-42}$ Results from our systematic review suggest that obstetric outcomes are similar, 
irrespective of the timing of defibulation; however, our related systematic review on women's motivation for and experience with surgical interventions found that defibulation in relation to childbirth is perceived as facilitating an easier birth, and women prefer to have the procedure performed during labour, rather than antenatally. ${ }^{43}$ Thus, our findings support the WHO guideline, which recommends that women should be consulted on their preferences for the timing of defibulation. ${ }^{7}$

Although the WHO guideline developers mentioned clitoral reconstruction, they issued no recommendations because of 'safety concerns'. ' Indeed, although it appears that postoperative complications for defibulation and reconstruction are comparable and minor, there is cause for concern with regards to harm from reconstruction. It is disquieting that studies on reconstructive surgery indicate a worsening in sexuality-related outcomes, in the $2-22 \%$ range.

\section{Conclusion}

Women seeking defibulation and reconstructive surgery should be informed about the scarcity of evidence on the benefits and harms of these procedures, in the short- and long-term, for most outcomes. Furthermore, although there are no or few indications of harm from defibulation, reconstruction may lead to a worsening in sexuality-related outcomes for as many as one in five women. Results from another systematic review document that most women appear to be satisfied with defibulation and reconstruction. ${ }^{43}$ Still, women's experiences with reparative surgeries are mixed. About one in ten women perceive their defibulated genitalia as more ugly, and with respect to reconstruction, a third of women are dissatisfied with or perceive a worsening in the aesthetic look. Thus, women who consider therapeutic surgeries should receive not just information about the options available to them, but what they can realistically expect with regard to benefits, harm, and satisfaction, including genital appearance.

Because the demand for care of FGM/C-related complications will remain for decades to come, and there is a general lack of technical knowledge and cultural competency in caring for women with $\mathrm{FGM} / \mathrm{C}$, ${ }^{4}$ there is a need for pre-service as well as in-service healthcare provider training programmes. There is some evidence that healthcare providers are interested in education on $\mathrm{FGM} / \mathrm{C},{ }^{45}$ and promising educational programmes on defibulation and repair are available. $^{46}$

This systematic review has identified the need for additional research on the benefits and harms of care interventions for women who live with FGM/C. There is a particular need for prospective, controlled studies - preferably RCTs - that investigate the range of patient-important outcomes. The methodological limitations of existing research show that future studies should recruit larger samples, have long-term follow-up, apply clear inclusion criteria, and use standardised data collection methods, and validated and reliable instruments.

\section{Disclosure of interests}

None declared. Completed disclosure of interests form available to view online as supporting information.

\section{Contribution to authorship}

RB conceptualised the study, wrote the protocol, screened the literature, extracted the data, performed the risk-of-bias assessment, analysed the data, and wrote the manuscript. ST contributed to the protocol, screened the literature, checked the data extraction and risk-of-bias assessment, assisted with data analysis, and co-wrote the manuscript. MS, IS, and SV contributed to the protocol, screened the literature, and commented on the manuscript.

\section{Details of ethics approval}

This is a systematic review and no ethics approval was necessary.

\section{Funding}

This research had no specific funding.

\section{Acknowledgement}

We are grateful for the superb assistance of information search specialist Sari Ormstad.

\section{Supporting Information}

Additional Supporting Information may be found in the online version of this article:

Figure S1. Meta-analysis of previous defibulation versus antenatal defibulation on the outcome caesarean section.

Table S1. Characteristics of included studies, by treatment $(k=62)$.

Table S2. References of included studies, in alphabetical order $(k=62)$.

Table S3. Studies excluded after full-text screening, with reason for exclusion $(k=70)$.

Table S4. Methodological quality assessment of included studies.

Table S5. Study outcomes and effect estimates of defibulation versus no defibulation for women with FGM/C.

Table S6. Study outcomes and effect estimates of antepartum defibulation versus intrapartum defibulation for women with FGM/C.

Table S7. Study outcomes and effect estimates of reconstruction (surgery-related complications and appearance) for women with FGM/C. 
Berg et al.

Table S8. GRADE 'summary of findings' table for the comparison defibulation versus no defibulation.

Table S9. GRADE 'summary of findings' table for the comparison antepartum defibulation versus intrapartum defibulation.

Table S10. GRADE 'summary of findings' table for the comparison previous defibulation versus antenatal defibulation.

Appendix S1. Search strategy.

Video S1. Author insights.

\section{References}

1 WHO. Eliminating female genital mutilation: An interagency statement. 2008 [www.unfpa.org/webdav/site/global/shared/docume nts/publications/2008/eliminating_fgm.pdf]. Accessed 15 December 2016

2 Berg RC, Underland V, Odgaard-Jensen J, Fretheim A, Vist GR. Effects of female genital cutting on physical health outcomes: a systematic review and meta-analysis. BMJ Open 2014;4:e006316.

3 Berg RC, Denison E. Does female genital mutilation/cutting (FGM/C) affect women's sexual functioning? A systematic review of the sexual consequences of FGM/C. Sex Res Soc Pol 2012;9:41-56.

4 Berg RC, Odgaard-Jensen J, Fretheim A, Underland V, Vist E. An updated systematic review and meta-analysis of the obstetric consequences of female genital mutilation/cutting. Obstet Gynecol Int 2014:542859.

5 Adam T, Bathija $H$, Bishai $D$, Bonnenfant $Y-T$, Darwish $M$ Huntingtona $D$, et al. Estimating the obstetric costs of female genital mutilation in six African countries. Bull World Health Organ 2010;88:281-8.

6 UNFPA-UNICEF. UNFPA-UNICEF joint programme on female genital mutilation/cutting: accelerating change. 2014 [www.unfpa.org/ publications/unfpa-unicef-joint-programme-female-genital-mutila tioncutting-accelerating-change]. Accessed 5 December 2016.

7 WHO. WHO guidelines on the management of complications from female genital mutilation. 2016 [www.who.int/reproductivehea Ith/topics/fgm/management-health-complications-fgm/en/]. Accessed 15 December 2016

8 Waters J. New de-infibulation clinic for Royal Women's in Melbourne. Aust Nurs J 2011;18:39.

9 Dominguez L, Hopkins K, Mutalak O, Harish S, Nalabanda A, Osman S, et al. Female genital mutilation (FGM)-providing a holistic approach and challenging taboos in a sexual health setting. Sex Transm Infect 2012;88:P153.

10 Albert J. 12 months later. Midwives 2010;13:40-1.

11 Paterson LQP, Davis SN, Binik YM. Les mutilations genitales feminines et l'orgasme avant et apres reparation chirurgicale. Sexologies 2012;21:21-8.

12 Abdulcadir J, Roderiguez MI, Say L. A systematic review of the evidence on clitoral reconstruction after female genital mutilation cutting. Int J Gynaecol Obstet 2015;129:93-7.

13 Foldès $P$, Cuzin $B$, Andro $A$. Reconstructive surgery after female genital mutilation: a prospective cohort study. Lancet 2012;380:134-41.

14 NHS. Clinical commissioning policy statement: genital surgery to improve clitoral sensation for women who have undergone female genital mutilation. 2015 [www.england.nhs.uk/commissioning/wpcontent/uploads/sites/12/2015/01/e10-surgcl-trt-fgm.pdf]. Accessed 15 December 2016
15 Higgins JPT, Green S. Cochrane Handbook for Systematic Reviews of Interventions. Version 5.1.0 ed. The Cochrane Collaboration, 2011.

16 Guyatt G, Rennie D, Mead MO, Cook DJ. User's Guides to the Medical Literature: A Manual for Evidence-Based Clinical Practice, 3rd edn. New York, NY: The McGraw-Hill Companies, Inc, 2015.

17 Guyatt GH, Oxman AD, Schunemann HJ, Tugwell P, Knottnerus A. GRADE guidelines: a new series of articles in the Journal of Clinical Epidemiology. J Clin Epidemiol 2011;64:380-2.

18 Balshem $H$, Helfand $M$, Schünemann $H J$, Oxman $A D$, Kunz $R$, Brozek J, et al. GRADE guidelines: 3. Rating the quality of evidence. J Clin Epidemiol 2011;64:401-6.

19 Abdulcadir J, Dugerdil A, Yaron M, Irion O, Boulvain M. Obstetric care of women with female genital mutilation attending a specialized clinic in a tertiary center. Int J Gynaecol Obstet 2016:132:174-8.

20 Albert J, Bailey E, Duaso M. Does the timing of deinfibulation for women with type 3 female genital mutilation affect labour outcomes? Br J Midwifery 2015;23:430-7.

21 Paliwal P, Ali S, Bradshaw S, Hughes A, Jolly K. Management of type III female genital mutilation in Birmingham, UK: a retrospective audit. Midwifery 2014;30:282-8.

22 Raouf SA, Ball T, Hughes A, Holder R, Papaioannou S. Obstetric and neonatal outcomes for women with reversed and non-reversed type III female genital mutilation. Int Gynaecol Obstet 2011;113: 171-3.

23 Bikoo M, Davies M, Richens $\mathrm{Y}$, Cerighton S. Female genital mutilation: a growing challenge for midwives in the $\mathrm{UK} . \mathrm{Br} J$ Midwifery 2006;14:403-5.

24 Minsart AF, N'guyen TS, Ali Hadji R, Caillet M. Maternal infibulation and obstetrical outcome in Djibouti. J Matern Fetal Neonatal Med 2015;28:1741-6.

25 Thabet SAA, Thabet ASMA. Defective sexuality and female circumcision: the cause and the possible management. I Obstet Gynaecol Res 2003;29:12-9.

26 Foldès $P$, Louis-Sylvestre $C$. Résultats de la réparation chirurgicale $d u$ clitoris après mutilation sexuelle: 453 cas. Gynecol Obstet Fertil 2006;34:1137-41.

27 Hussen AO, Catania L. Deinfibulazione terapeutica. Ginecologo 2006;1:72-5.

28 Krause E, Brandner S, Mueller MD, Kuhn A. Out of Eastern Africa: defibulation and sexual function in woman with female genital mutilation. J Sex Med 2011;8:1420-5.

29 Merckelbagh HM, Nicolas MN, Piketty MP, Benifla JL. Évaluation d'une prise en charge multidisciplinaire chez 169 patientes excises demandeuses d'une chirurgie réparatrice. Gynecol Obstet Fertil 2015:43:633-9.

30 Ouedraogo CMR, Madzoy S, Toure B, Ouedraogo A, Ouedraogo S, Lankoande J. Pratique de la chirurgie plastique reconstructrice du clitoris apres mutilations genitales au Burkina Faso. A' propos de 94 cas. Ann Chir Plast Esthet 2013;58:208-15.

31 Sirigatti S, Catania L, Simone S, Casale S, Hussen AO. Preliminary research into the psycho-sexual aspects of the operation of definbulation. In: Denniston GC, Grassiovaro Gallo P, Hodges FM, Milos MF, Viviani $F$, editors. Bodily Integrity and the Politics of Circumcision. New York, NY: Springer; 2006. pp 123-32.

32 Catania L, Abdulcadir O, Puppo V, Verde JB, Abdulcadir J, Abdulcadir D. Pleasure and orgasm in women with Female Genital Mutilation/Cutting (FGM/C). J Sex Med 2007;4:1666-78.

33 Nour NM, Michels KB, Bryant AE. Defibulation to treat female genital cutting: effect on symptoms and sexual function. Obstet Gynecol 2006; 108:55-60 
34 Abdulcadir J, Rodriquez MI, Petignat P, Say L. Clitoral reconstruction after female genital mutilation/cutting: case studies. J Sex Med 2015;12:274-81.

35 Lopez-Olmos J. Mutilación genital y reconstruccion del clitoris. Clin Invest Ginecol Obstet 2016;43:92-6.

36 Quilichini J, Burin Des Roziers B, Daoud G, Cartier S. Chirurgie réparatrice du clitoris après excision rituelle. Ann Chir Plast Esthet 2011;56:74-9.

37 Abramowicz A, Oden S, Dietrich G, Marpeau L, Resch B. Évaluation des résultats anatomoques, fonctionnels et identitaires après transposition du clitoris chez 30 patientes. J Gynecol Obstet Biol Reprod 2016;45:963-71.

38 Sigurjonsson $\mathrm{H}$, Jordal $\mathrm{M}$, Lundgren TK. Reconstructive surgery after female genital mutilation. Int J Gynaecol Obstet 2015;131 (Suppl 5): E318-9.

39 Balogun OO, Hirayama F, Wariki WMV, Koyanagi A, Mori R. Interventions for improving outcomes for pregnant women who have experienced genital cutting. Cochrane Database Syst Rev 2013; (2):CD009872

40 Royal College of Obstetricians and Gynaecologists. Female genital mutilation and its management. Green-top Guideline No. 53. 2015 [www.rcog.org.uk/en/guidelines-research-services/guidelines/gtg53/]. Accessed 15 December 2016.

41 Amman C, Cotting A, Hanselmann V, Held P, Hohlfeld P, Hollinger $E$, et al. Female genital mutilations: Swiss recommendations for health professionals. 2005 [www.sggg,ch/fileadmin/user_upload/ Dokumente/3_Fachinformationen/2_Guidelines/Fr/Mutilations_genita le_feminines_2005]. Accessed 15 December 2016.

42 Royal College of Nursing. Female genital mutilation. 2015 [www.rc n.org.uk/professional-development/publications/pub-005447]. Accessed 1 March 2017.

43 Berg RC, Taraldsen S, Said MA, Sørbye IK, Vangen S. Reasons for and experiences with surgical interventions for female genital mutilation/cutting (FGM/C): a systematic review. J Sex Med 2017; 14:977-90.

44 Dawson A, Turkmani S, Fray S, Nanayakkara S, Varol N, Homer C. Evidence to inform education, training and supportive work environments for midwives involved in the care of women with female genital mutilation: a review of global experience. Midwifery 2015;31:229-38.

45 Lane J, Johnson-Agbakwu C, Cole EC. Knowledge of female genital cutting/circumcision and the influence on practice: a survey of obstetric and gynecological healthcare providers in the United States. J Sex Med 2014;11:224.

46 Jacoby SD, Smith A. Increasing certified nurse-midwives' confidence in managing the obstetric care of women with female genital mutilation/cutting. J Midwifery Womens Health 2013;58: 451-6. 